\title{
THE NITROGEN SPECTRA OF WN STARS: THE WN6 "STANDARD” STAR HD 192163 (WR136)
}

\author{
U. WESSOLOWSKI ${ }^{1}$, W.-R. HAMANN ${ }^{1}$, W. SCHMUTZ ${ }^{2}$ \\ 1 Institut für Theoretische Physik und Sternwarte der Universität \\ Olshausenstraße 40, D-2300 Kiel 1, FRG \\ 2 Joint Institute for Laboratory Astrophysics \\ University of Colorado, Boulder, CO 80309-0440, USA
}

Hitherto our quantitative analyses of WR spectra [2][5] have been based on pure-helium models [1][6]. Now we further improved our non-LTE calculations by including a complex model atom of nitrogen (90 energy levels, 351 line transitions; with low-temperature dielectronic recombination) into our model atmospheres in order to synthesize adequately the spectra of WN subtypes (Wessolowski et al., in preparation). Together with the nitrogen (the most important "metal" in WN atmospheres), we introduced an improved temperature structure into our model calculations [3], now accounting for non-grey radiative equilibrium instead of the grey approximation applied so far. Moreover we took into account the line overlap of the considered elements (here: helium, nitrogen) and also their blanketing effects on the continuous radiation field.

Theoretical line profiles of nitrogen are compared with the observed spectrum of HD 192163 (alias WR136), a well-known WN6 "standard" star (Table 1). Most of the equivalent widths of the observed nitrogen lines can be reproduced within a factor of 2 to 3, but only by two slightly different models (Model 1: $T_{*}=50 k K$ and $R_{*}=6.0 R_{\odot}$, Model 2: $T_{*}=60 k K$ and $R_{*}=5.5 R_{\odot}$; both models with $\log \left[\dot{M} /\left(M_{\odot} y r^{-1}\right)\right]=-3.85, v_{\infty}=$ $1700 \mathrm{~km} / \mathrm{s}$ and a nitrogen abundance $\beta_{N}=1.5 \%$ by mass).

TABLE 1. Equivalent widths $[\AA]$ of nitrogen lines for HD 192163 (WN6)

\begin{tabular}{l|c|cccc|ccc}
\hline nitrogen & N III & \multicolumn{4}{|c|}{ N IV } & \multicolumn{3}{c}{ N V } \\
lines & $\lambda 4640$ & $\lambda 1486$ & $\lambda 1718$ & $\lambda 3480$ & $\lambda 4058$ & $\lambda 1240$ & $\lambda 4610$ & $\lambda 4944$ \\
\hline Observed & 79 & 29 & 34 & 60 & 38 & 10 & 10 & 3 \\
Model 1 & 30 & 58 & 10 & 12 & 28 & 3 & 1 & 2 \\
Model 2 & 13 & 81 & 15 & 41 & 43 & 11 & 1 & 3 \\
\hline
\end{tabular}

Altogether these results confirm the tendencies of Hillier's cool wind model for the WN5 star HD 50896 [4]. Remaining problems may be attributed to the very complex model atom and minor uncertainties in the stellar parameters and the temperature structure.

\section{References:}

[1] Hamann, W.-R., Schmutz, W.: 1987, Astron. Astrophys. 174, 173

[2] Hamann, W.-R., Schmutz, W., Wessolowski, U.: 1988, Astron. Astrophys. 194, 190

[3] Hamann, W.-R., Wessolowski, U.: 1990, Astron. Astrophys. 227, 171

[4] Hillier, D.J.: 1988, Astrophys. J. 327, 822

[5] Schmutz, W., Hamann, W.-R., Wessolowski, U.: 1989, Astron. Astrophys. 210, 236

[6] Wessolowski, U., Schmutz, W., Hamann, W.-R.: 1988, Astron. Astrophys. 194, 160 